\title{
HAADF-STEM and Super-XTM XEDS Tomography of Complex Nano-scale Precipitates in a High Entropy Alloy, AlMo0.5NbTa ${ }_{0.5}$ TiZr
}

\author{
J.K. Jensen ${ }^{1}$, J.M. Sosa ${ }^{1}$, D.E. Huber ${ }^{1,2}$, G.B. Viswanathan ${ }^{1}$, R.E.A. Williams ${ }^{1,2}$, H.L. Fraser ${ }^{1}$ \\ 1. Center for the Accelerated Maturation of Materials, The Ohio State University, Columbus, OH \\ 2. Center for Electron Microscopy and Analysis, The Ohio State University, Columbus, OH
}

High entropy alloys (HEAs) are a relatively new class of materials garnering a great deal of attention due to their remarkable balance of properties, including high strength, toughness, ductility, and corrosion resistance. In contrast to conventional alloy systems, HEAs are based on 4 or more principal elements with near equimolar concentrations and tend to have simple microstructures due to the preferential formation of solid solution phases attributed to the high configurational entropy of mixing [1]. HEAs appear to offer new pathways to lightweighting in structural applications, new alloys for intermediate and elevated temperature components, and new magnetic materials. However, to realize this potential requires considerable alloy development that will rely on application of integrated computational materials (science and) engineering (ICME). The development of accurate computational models to predict alloy performance requires a detailed knowledge of the morphology and interconnectedness of microstructural features. Due to spinodal decomposition, these alloys often consist of a mixture of ordered and disordered phases on the nano-scale, and because of the compositional and structural complexity, it is desirable to characterize the morphology as well as the chemical nature of the precipitates for inclusion in ICME models.

Electron tomography is a powerful characterization technique capable of resolving microstructural features at length scales as small as a few nanometers when coupled with the high spatial resolution available with modern scanning transmission electron microscopes (STEM). For STEM tomography specifically, an electron beam is transmitted through a sample at incremental degrees of rotation and images are recorded at each tilt. The images are then processed to reconstruct a three-dimensional representation of the area of interest. Tomographic reconstructions, in general, may be produced by any set of images collected though an angle of rotation with the quality of the reconstruction depending directly on the angular tilt range collected. A previous tomography study on the AlMo ${ }_{0.5} \mathrm{NbTa} 0.5 \mathrm{TiZr}$ HEA was reconstructed from only high angle annular dark-field (HAADF) STEM images and successfully resolved the nano-scale precipitates in the microstructure [2]. While HAADF contrast was sufficient to resolve and segment the nano-scale precipitates in this HEA, the reconstruction provided only morphological information and no compositional data. In order to obtain a novel perspective of the 3D elemental segregation in the HEA, this paper will present the use of Super- $\mathrm{X}^{\mathrm{TM}}$ energy dispersive $\mathrm{X}$ ray spectroscopy (XEDS) spectral images (SI) utilized in conjunction with HAADF-STEM images for an improved tomographic reconstruction of the AlMo ${ }_{0.5} \mathrm{NbTa}_{0.5} \mathrm{TiZr}$ alloy.

Rather than using a conventional thin foil STEM sample geometry, a tapered needle-like sample was created using a FEI Helios ${ }^{\mathrm{TM}}$ DualBeam FIB. This geometry was selected to eliminate thickness variations that occur with larger degrees of tilting with flat samples as well as to allow for $360^{\circ}$ tilting using a Fischione 2050 on-axis rotation tomography holder. A tilt series of HAADF-STEM micrographs and Super-X ${ }^{\mathrm{TM}}$ XEDS SI were collected using a FEI Titan ${ }^{\mathrm{TM}}$ 60-300 STEM at $1^{\circ}$ increments along the longitudinal axis of the sample. XEDS SI were collected for 300s with a dwell time/pixel of $20 \mu$ s. All data was aligned, segmented and reconstructed using MIPAR ${ }^{\mathrm{TM}}$ image processing software [3]. A 
weighted back projection was then applied using the TomoJ plugin in ImageJ. Image processing produced a three-dimensional reconstruction of the microstructure based off the HAADF images and the XEDS signals. Figure 1(a) shows the rendered 3D volume of the AlMo0.5NbTa0.5 TiZr HEA, revealing a periodic microstructure composed of cuboidal and platelet precipitates. The reconstructions of the $\mathrm{Zr}$ and Ta EDX maps, shown in Figure 1(b) and 1(c) respectively, illustrate the segregation of $\mathrm{Zr}$ to the ordered B2 matrix and Ta to the disordered cuboidal and platelet precipitates. This 3D compositional variation was inherently difficult to observe through traditional 2D characterization, specifically the case of thin platelet in a stacked morphology where precipitate/matrix projection artifacts prevent local 2D compositional measurements.

\section{References:}

[1] J.W. Yeh et al, Materials Science Forum 560 (2007), p. 1.

[2] J.M. Sosa et al, Materials Science and Technology (2015). Submitted

[3] J.M. Sosa et al, Integrating Materials and Manufacturing Innovation 3 (2014), p. 18

[4] The authors acknowledge funding and support from the DAGSI program and the Air Force Research Lab (AFRL) in Dayton, OH. Thanks to Fischione for use of the 2050 on-axis tomography holder.

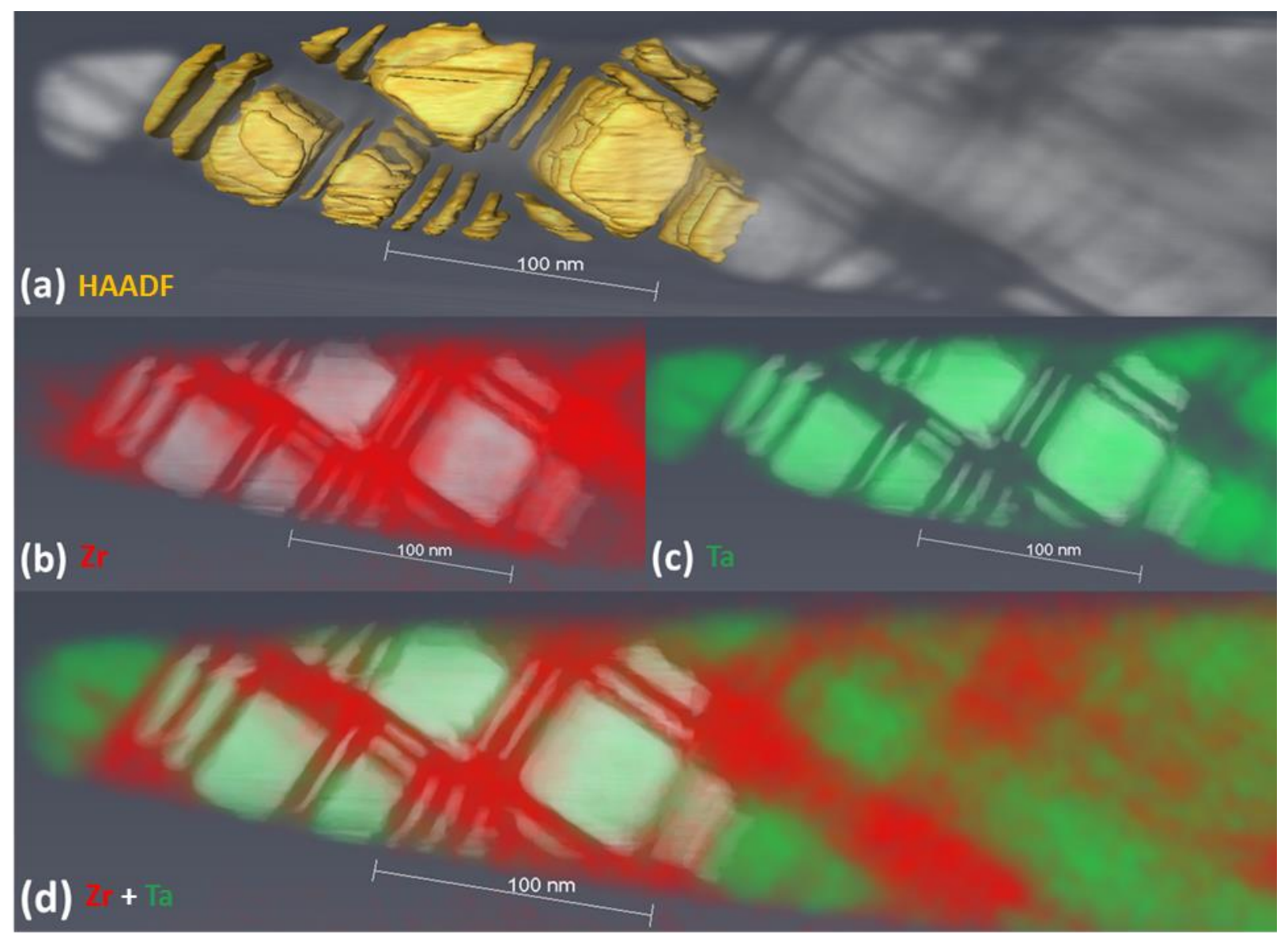

Figure 1. (a) 3D HAADF reconstruction of $\mathrm{AlMo}_{0.5} \mathrm{NbTa}_{0.5} \mathrm{TiZr}$ tapered needle with segmented cuboidal and platelet precipitates superimposed with Super- ${ }^{\mathrm{TM}}$ XEDS tomographic reconstructions of (b) Ta and (c) Zr elemental maps. (d) Composite reconstruction with $\mathrm{Ta}$ (red), Zr (green), and HAADF (white) 3D renderings. 